\title{
Cycles of mitochondrial energization driven by the ultradian clock in a continuous culture of Saccharomyces cerevisiae
}

\author{
David Lloyd ${ }^{1}$ L. Eshantha J. Salgado, ${ }^{1}$ Michael P. Turner, ${ }^{1}$ \\ Marc T. E. Suller ${ }^{1}$ and Douglas Murray ${ }^{2}$
}

Author for correspondence: David Lloyd. Tel: +44 29 20874772. Fax: +44 2920874305. e-mail: Lloydd@cf.ac.uk

\footnotetext{
1 Microbiology (BIOSI 1, Main Building), Cardiff University, PO Box 915, Cardiff CF10 3TL, Wales, UK

2 School of Applied Science, University of the South Bank, 103 Borough Road, London SET OAA, UK
}

\begin{abstract}
A continuous culture of Saccharomyces cerevisiae IFO 0233, growing with glucose as the major carbon and energy source, shows oscillations of respiration with a period of $48 \mathrm{~min}$. Samples taken at maxima and minima indicate that (i) periodic changes do not occur as a result of carbon depletion, (ii) intrinsic differences in respiratory activity occur in washed organisms and (iii) a respiratory inhibitor accumulates during respiratory oscillations. Plasma membrane and inner mitochondrial membranes generate transmembrane electrochemical potentials; changes in these can be respectively assessed using anionic or cationic fluorophores. Thus flow cytometric analyses indicated that an oxonol dye [DiBAC 4 (3); bis(1,3-dibutylbarbituric acid)trimethine oxonol] was excluded from yeasts to a similar extent (in $>98 \%$ of the population) at all stages, showing that the plasma membrane potential was maintained at a steady value. However, uptake of Rhodamine 123 was greatest at that phase characterized by a low respiratory rate. Addition of uncouplers of energy conservation [CCCP (m-chlorocarbonylcyanide phenylhydrazone) or S-13 (5-chloro-3-t-butyl-2-chloro-4' ${ }^{1}$-nitrosalicylanilide)] to the continuous cultures increased the respiration, but had only a transient effect on the period of the oscillation. Electron microscopy showed changes in mitochondrial ultrastructure during the respiratory oscillation. At low respiration the cristae were more clearly defined due to swelling of the matrix; this corresponds to the 'orthodox' conformation. When respiration was high the mitochondrial configuration was 'condensed'. It has been shown previously that a temperature-compensated ultradian clock operates in S. cerevisiae. It is proposed that mitochondria undergo cycles of energization in response to energetic demands driven by this ultradian clock output.
\end{abstract}

Keywords: mitochondria, redox regulation, NADH, cytochrome, ultradian clock

\section{INTRODUCTION}

Continuous cultures of Saccharomyces cerevisiae display a temperature-compensated rhythm of respiration which can be continuously monitored over extended periods (months) by measurement of dissolved $\mathrm{O}_{2}$ (Satroutdinov et al., 1992). This is one output of many

Abbreviations: CCCP, $m$-chlorocarbonylcyanide phenylhydrazone; $\mathrm{DiBAC}_{4}(3)$, bis(1,3-dibutylbarbituric acid)trimethine oxonol; S-13, 5-chloro3-t-butyl-2-chloro-4'-nitrosalicylanilide; DOT, dissolved $\mathrm{O}_{2}$ tension; SHAM, salicylhydroxamic acid. driven by the ultradian clock (Murray et al., 2001), which has, in this yeast strain, a characteristic period of about $40 \mathrm{~min}$ over the range $25-35^{\circ} \mathrm{C}$ (temperature quotient; $\left.\mathrm{Q}_{10}=1 \cdot 07\right)$. Other cyclically varying outputs include sulphate uptake (Sohn \& Kuriyama, 2001), ethanol production (Keulers et al., 1996a), glutathione to oxidized glutathione dimer interconversion (Murray et al., 1999) as well as intracellular redox states, as indicated by continuous in vivo monitoring of NADH fluorescence (Murray et al., 1998a, b), and calculated values of intracellular $\mathrm{pH}$ (Satroutdinov et al., 1992). It is proposed under the highly acid culture conditions employed that metabolic synchrony is elicited by per- 


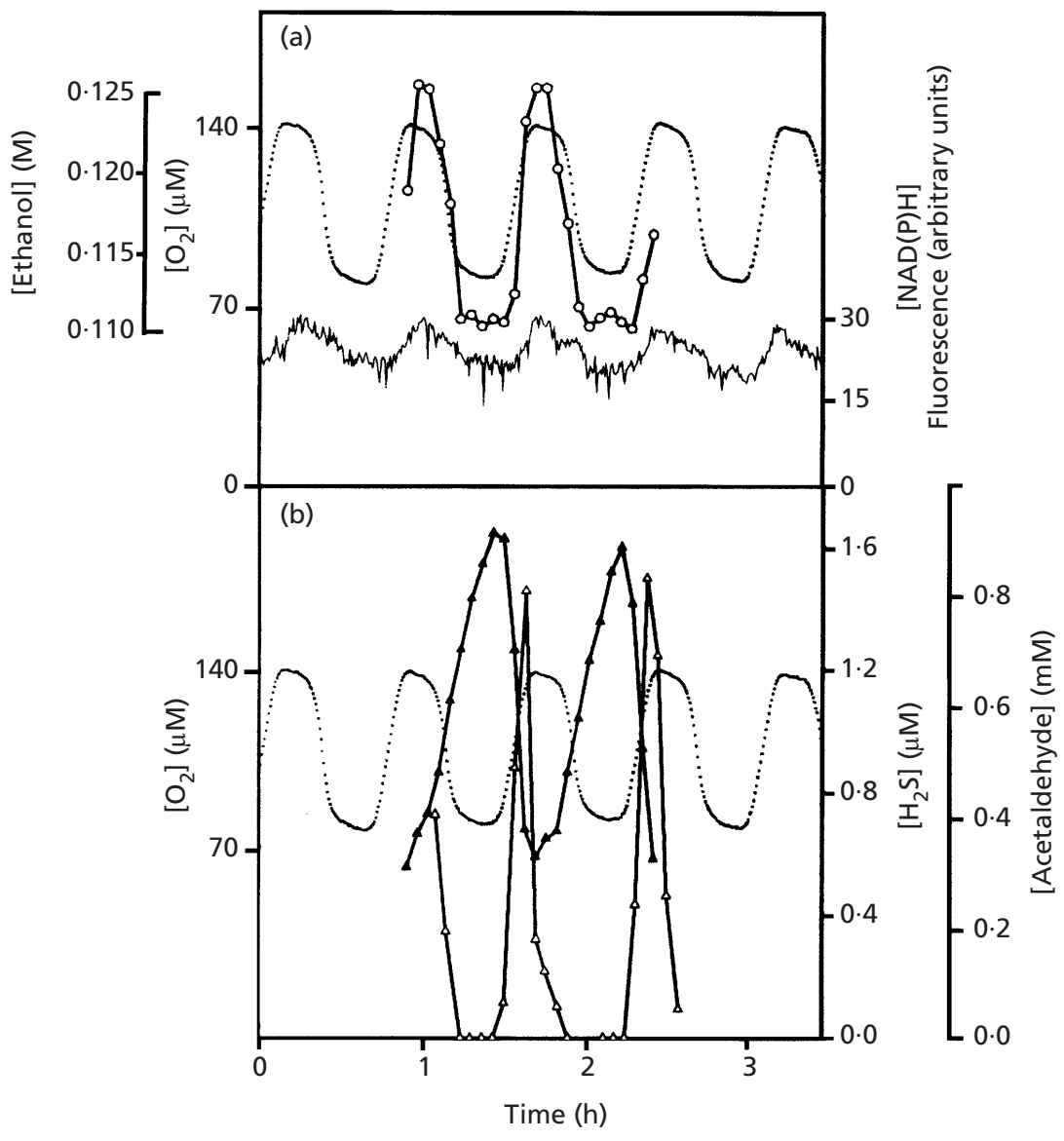

Fig. 1. Oscillatory outputs of the ultradian clock in a continuous culture of $S$. cerevisiae. (a) DOT (dashed line), NADH fluorescence (continuous trace), both measured directly, simultaneously and continuously, and ethanol concentration ( $O$ ). (b) DOT (dashed line), acetaldehyde $(\boldsymbol{\Delta})$ and $\mathrm{H}_{2} \mathrm{~S}(\triangle)$. iodic release of $\mathrm{H}_{2} \mathrm{~S}, 180^{\circ}$ out of phase with acetaldehyde accumulation (Sohn et al., 2000).

The oscillatory behaviour of respiration is not dependent on glycolytic oscillations as is evidenced by its occurrence with a similar persistence and temperaturecompensated period during growth with ethanol (Keulers et al., 1996a, b; Murray et al., 2001). Glycolytic oscillations are different in that they are characterized by shorter periods (about 1 to $5 \mathrm{~min}$ ), are highly damped and not temperature-compensated (Lloyd \& Edwards, 1984, 1987).

The aim of the present work is to study changes in mitochondrial structure and function in situ during respiratory oscillations. We conclude that growth is accompanied by in vivo respiratory control (ADPacceptor control) involving cycles of energization and de-energization of mitochondria (Chance \& Williams, 1956; Luzikov, 1984; Lloyd, 1974) in response to energetic demand.

\section{METHODS}

Organisms and cultures. Saccharomyces cerevisiae strain IFO 0233 was used (Keulers et al., 1996a). The medium consisted of glucose monohydrate $\left(22 \mathrm{~g} \mathrm{l}^{-1}\right),\left(\mathrm{NH}_{4}\right)_{2} \mathrm{SO}_{4} \quad\left(5 \mathrm{~g} \mathrm{l}^{-1}\right)$, $\mathrm{KH}_{2} \mathrm{PO}_{4}\left(2 \mathrm{~g} \mathrm{l}^{-1}\right), \quad \mathrm{MgSO}_{4} .7 \mathrm{H}_{2} \mathrm{O} \quad\left(0.5 \mathrm{~g} \mathrm{l}^{-1}\right), \mathrm{CaSO}_{4} .5 \mathrm{H}_{2} \mathrm{O}$
(0.005 $\left.\mathrm{g} \mathrm{l}^{-1}\right), \mathrm{MnCl}_{2} \cdot 4 \mathrm{H}_{2} \mathrm{O}\left(0 \cdot 001 \mathrm{~g} \mathrm{l}^{-1}\right)$, yeast extract (Difco; $\left.1 \mathrm{~g} \mathrm{l}^{-1}\right)$ and $70 \%(\mathrm{v} / \mathrm{v}) \mathrm{H}_{2} \mathrm{SO}_{4}\left(1 \mathrm{ml} \mathrm{l}^{-1}\right)$. Sigma Antifoam A was used at $1 \mathrm{ml} \mathrm{l}^{-1}$.

Continuous culture was carried out as described previously (Satroutdinov et al., 1992). The fermenter (LH500; Adaptive Biosystems) was operated with a stirrer rate of 900 r.p.m., a working volume of 0.81 , an airflow rate of $180 \mathrm{ml} \mathrm{min}^{-1}$ and a dilution rate of $0.085 \mathrm{~h}^{-1}$. The temperature was $30{ }^{\circ} \mathrm{C}$ and the $\mathrm{pH}$ was controlled at 3.4 by the addition of $2.5 \mathrm{M} \mathrm{NaOH}$. Dissolved $\mathrm{O}_{2}$ was monitored continuously using an Ingold $\mathrm{O}_{2}$ electrode. Experiments were conducted in continuous light. On line data acquisition was carried out using an in-house constructed PC-based system. Uncouplers of energy conservation [CCCP ( $m$-chlorocarbonylcyanide phenylhydrazone) and S-13 (5-chloro-3- $t$-butyl-2-chloro- $4^{1}$-nitrosalicylanilide)] were added as alcoholic solutions.

Cellular respiration. Organisms in growth medium were transferred directly to a closed $\mathrm{O}_{2}$ electrode system at $30^{\circ} \mathrm{C}$ (Rank) for measurements of respiration of the culture samples $(2 \mathrm{ml})$ taken at maxima and minima of dissolved $\mathrm{O}_{2}$ tension (DOT) in the oscillating state. Inhibitors $[\mathrm{Na}$ azide, SHAM (salicylhydroxamic acid)], or uncouplers (CCCP, S-13) (Hanstein, 1976) were added during the initial linear stage of $\mathrm{O}_{2}$ consumption. Where washed non-proliferating organisms were studied, they were centrifuged at 2000 r.p.m. (average $1000 \mathrm{~g}$ ) for $2 \mathrm{~min}$ at room temperature $\left(22^{\circ} \mathrm{C}\right.$ ) in a MSE Minor Centrifuge. Resuspension was in $4.5 \mathrm{ml} 50 \mathrm{mM}$ citric acid/ $\mathrm{Na}$ citrate buffer ( $\mathrm{pH} 3 \cdot 4$ ), followed by rapid recentrifugation in haematocrit tubes and resuspension to a final total 
volume of $0.5 \mathrm{ml}$ in the same buffer. Dilution with the same buffer $(1.5 \mathrm{ml})$ was followed by supplementation to a final concentration of $10 \mathrm{mM}$ glucose in the reaction mixture in the $\mathrm{O}_{2}$ electrode vessel. Some samples were force-aerated with sterile air at $30{ }^{\circ} \mathrm{C}\left(200 \mathrm{~cm}^{3} \mathrm{~min}^{-1}\right)$ to purge them of volatile fermentation products (e.g. acetaldehyde, $\mathrm{H}_{2} \mathrm{~S}$, ethanol, acetic acid) : the off-gas was led through a cold-trap kept at $77 \mathrm{~K}$ using liquid $\mathrm{N}_{2}$.

Measurement of $\mathrm{H}_{2} \mathbf{S}$ in the culture off-gas. Exit gas from the oscillating culture was allowed to impinge on a $\mathrm{AgNO}_{3}^{-}$impregnated test paper; the absorbance of the black deposit of $\mathrm{Ag}_{2} \mathrm{~S}$ was proportional to the $p\left(\mathrm{H}_{2} \mathrm{~S}\right)$ in the mobile gas phase. Calibration (Vogel, 1954) was with standard solutions of $\mathrm{Na}_{2} \mathrm{~S}$ brought to $\mathrm{pH} 3.4$ with $\mathrm{H}_{2} \mathrm{SO}_{4}$ and displacement at $30{ }^{\circ} \mathrm{C}$ with an air flow rate of $180 \mathrm{~cm}^{3} \mathrm{~min}^{-1}$ (identical to that used for aeration of the continuous culture).

Use of membrane-potential-sensitive fluorophores. For assessment of plasma-membrane electrochemical potential we used $1 \mu \mathrm{g} \mathrm{ml}^{-1}$ of an oxonol fluorophore, $\operatorname{DiBAC}_{4}(3)$ [bis(1,3dibutylbarbituric acid)trimethine oxonol] (Dinsdale et al., 1995). Incubation with organisms still suspended in their culture medium was for $30 \mathrm{~min}$ at room temperature $\left(22^{\circ} \mathrm{C}\right)$. For the inner mitochondrial membrane potential, Rhodamine $123\left(1 \mu \mathrm{g} \mathrm{ml}^{-1}\right)$ was employed under identical conditions (Chen, 1988). Flow cytometry was performed using a BectonDickinson cytometer. Excitation and emission wavelengths were $488 \mathrm{~nm}$ and $540 \mathrm{~nm}$, respectively, for both the $\mathrm{DiBAC}_{4}(3)$ - and Rhodamine 123-stained yeasts. For direct fluorimetric estimation of the latter, organisms were harvested at intervals from culture samples incubated with $1 \mu \mathrm{M}$ Rhodamine 123 using excitation at $540 \mathrm{~nm}$, emission at $580 \mathrm{~nm}$.

Redox state measurements. Samples for cytochrome spectra at high and low DOT $(3.8 \mathrm{ml}$ culture) were centrifuged at $1000 \mathrm{~g}$ for $2 \mathrm{~min}$ in a MSE Minor centrifuge using haematocrit tubes. Resuspension was to a volume of $0.2 \mathrm{ml}$ in 1.0 M mannitol. Reduced-oxidized cytochrome spectra were obtained at $77 \mathrm{~K}$ in a Unicam SP 1800 spectrophotometer, using Perspex cuvettes with a light path of 5 or $2 \mathrm{~mm}$. The oxidized sample was aerated and the reduced sample was kept anaerobic immediately before freezing. Samples were thawed and then reequilibrated to $77 \mathrm{~K}$. Spectra were obtained at a band-width of $2 \cdot 2 \mathrm{~nm}$ and with a scan rate of $2 \mathrm{~nm} \mathrm{~s}^{-1}$ over a spectral range of 400 to $620 \mathrm{~nm}$.

The redox state of the cellular $\mathrm{NAD}(\mathrm{P}) \mathrm{H} / \mathrm{NAD}^{+}$couple was monitored continuously in the culture using the method of Harrison \& Chance (1970) using a metabolite fluorimeter (Chance et al., 1975) directly on the fermentation vessel.

Electron microscopy. Samples were fixed as rapidly as possible by rapid sedimentation (Eppendorf Centrifuge, 14000 r.p.m., $30 \mathrm{~s}$ ) followed by mixing equal volumes with $0 \cdot 1 \mathrm{M}$ cacodylate buffer pH 6.9 containing $1 \%$ paraformaldehyde, $2 \%$ glutaraldehyde, $4 \%(\mathrm{v} / \mathrm{v})$ sucrose and $0.02 \% \mathrm{CaCl}_{2}$. After $1 \mathrm{~h}$ at $4{ }^{\circ} \mathrm{C}$, fixed organisms were sedimented and then resuspended in $1 \% \mathrm{OsO}_{4}$ at $4{ }^{\circ} \mathrm{C}$ for $1 \mathrm{~h}$. Dehydration by passage through a series of ethanol solutions (50,70 and $90 \%, 15 \mathrm{~min}$ each; $100 \%, 2 \times 30 \mathrm{~min}$ at room temp.). Infiltration with Spurr resin was overnight, followed by fresh resin for $8 \mathrm{~h}$ and polymerized overnight at $60^{\circ} \mathrm{C}$. Sections of $60-90 \mathrm{~nm}$ thickness were obtained using a LKB Ultramicrotome: staining with uranyl acetate was for $15 \mathrm{~min}$, followed by Reynold's lead citrate for $10 \mathrm{~min}$. The microscope was a JEOL 1210 transmission machine.

\section{RESULTS}

\section{Oscillatory metabolic changes in the continuous culture}

Fig. 1 a shows a typical section of the continuously monitored output from the $\mathrm{O}_{2}$ electrode measuring the DOT in the continuous culture. An autonomous wideamplitude excursion of the trace from $80 \mu \mathrm{M} \mathrm{O}_{2}$ at the respiratory maxima to $140 \mu \mathrm{M} \mathrm{O}_{2}$ at the respiratory minima and back occurred every $48 \mathrm{~min}$. Also shown (Fig. 1a) is the $\mathrm{NAD}(\mathrm{P}) \mathrm{H}$ fluorescence emission excited by the $366 \mathrm{~nm}$ line of a $\mathrm{Hg}$ arc lamp directly illuminating the culture and measured at $450 \mathrm{~nm}$. The redox state of the nicotinamide nucleotide pools oscillated with the same period as that of the respiratory oscillations, and maximum reduction (i.e. maximum fluorescence emis-
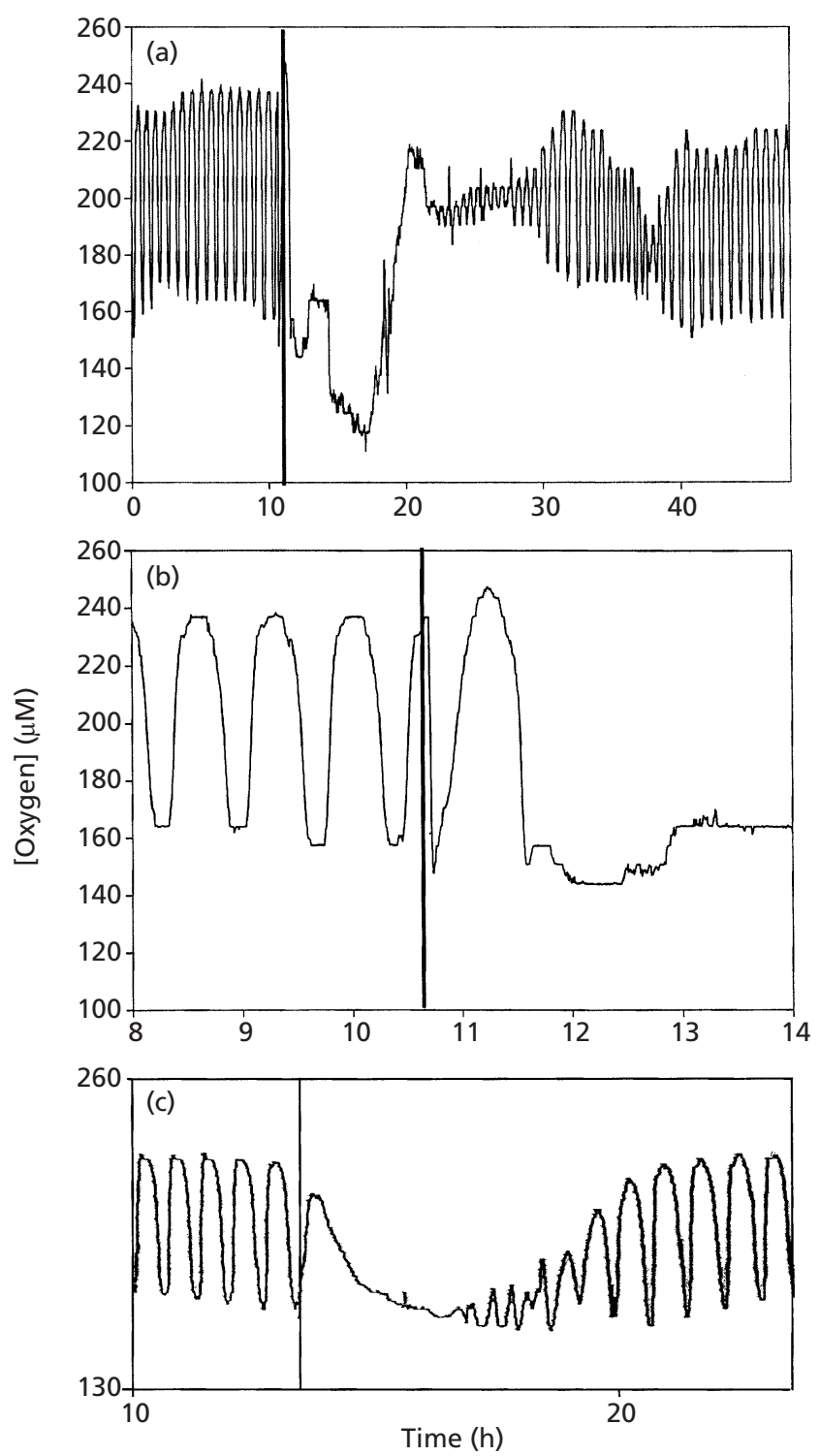

Fig. 2. Uncoupling energy conservation in a continuous culture of S. cerevisiae. At the solid vertical lines $10 \mu \mathrm{M}$ CCCP $(a, b)$ or $4 \mu \mathrm{M}$ S-13 (c) was injected aseptically. 
(a)

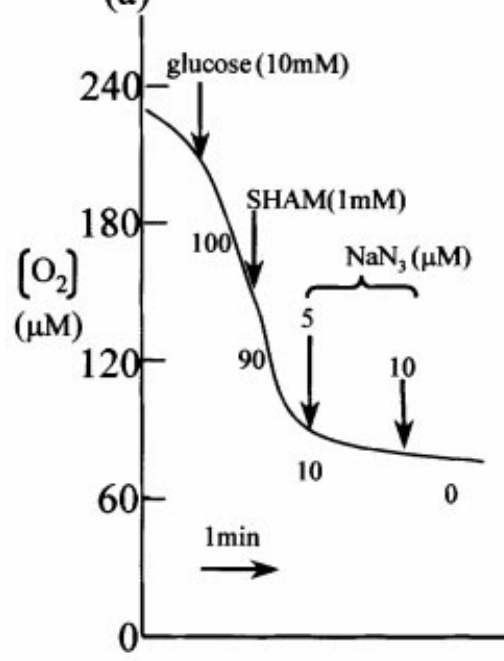

(b)

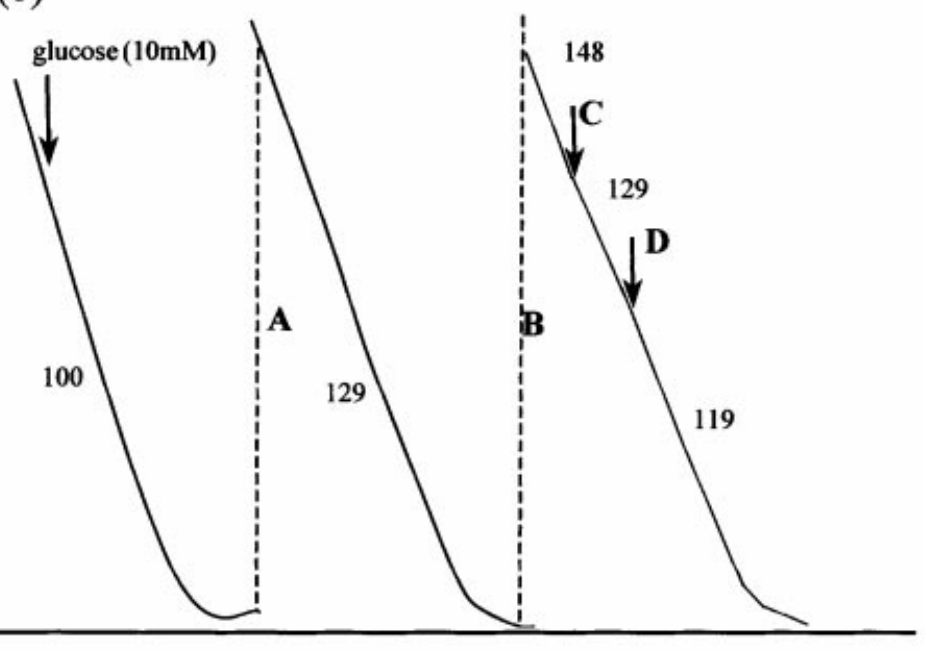

(c)

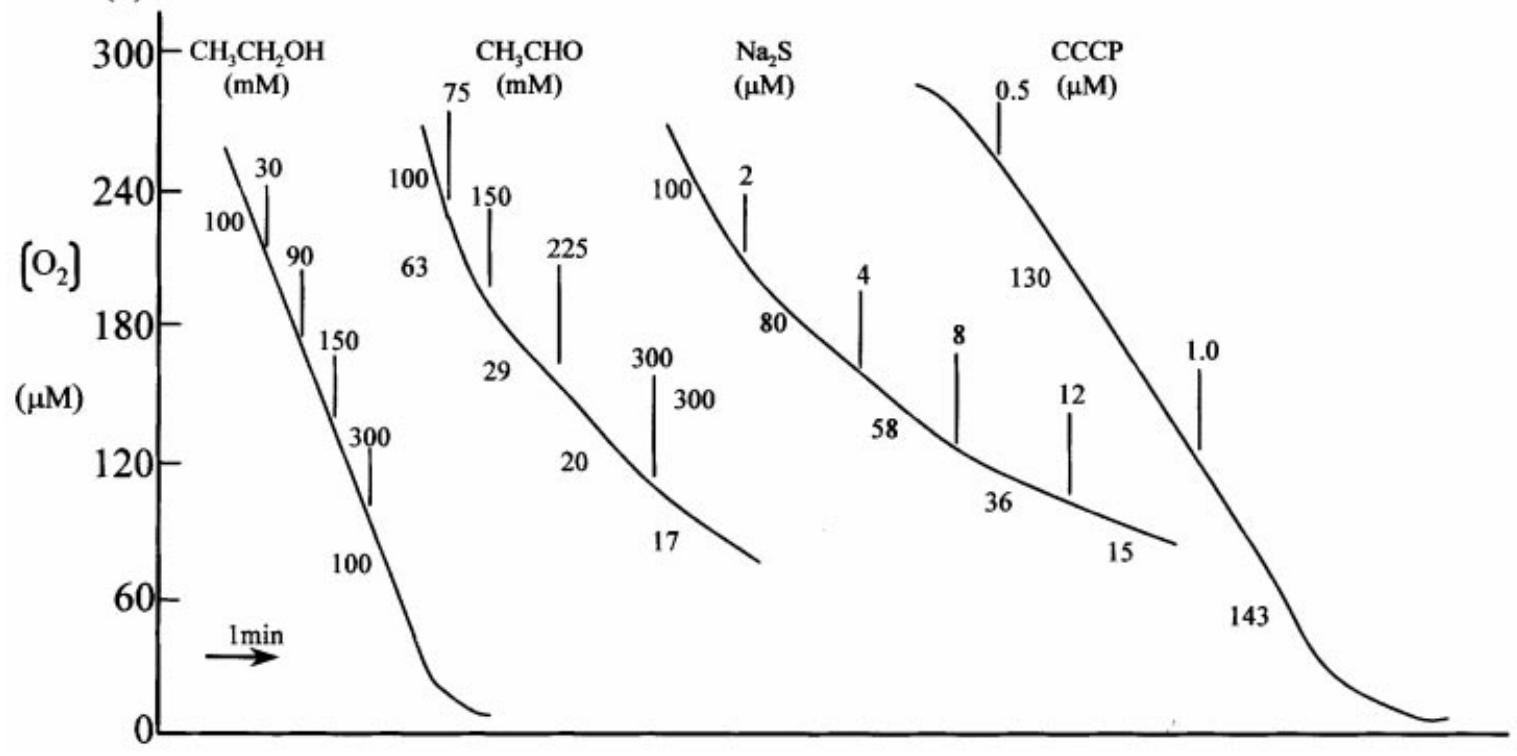

Fig. 3. Oxygen consumption of samples removed from the oscillating continuous culture of $S$. cerevisiae. (a) Washed nonproliferating organisms suspended in $50 \mathrm{mM}$ sodium citrate buffer (pH 3.4). (b) Culture was diluted $1+3$ with the same buffer. At $\mathbf{A}$ and $\mathbf{B}$ reaeration was for $5 \mathrm{~min}$; distillate was trapped at $77 \mathrm{~K}$ and added back at C and D. (c) Nonproliferating organisms incubated in the presence of $10 \mathrm{mM}$ glucose. Examples shown were all from phases of maximal respiration $\left[6 \mathrm{nmol} \mathrm{O}_{2} \min ^{-1}\left(10^{7} \text { yeast cells }\right)^{-1}\right]$; similar results were obtained with organisms from phases of minimal respiration $\left[4 \mathrm{nmol} \mathrm{O}_{2} \mathrm{~min}^{-1}\left(10^{7} \text { yeast cells }\right)^{-1}\right]$. Numbers shown on the traces indicate rates as a percentage of controls (untreated suspensions). Cell density in the oscillatory culture was $7 \times 10^{8}$ organisms $\mathrm{ml}^{-1}$. Traces shown are typical of results with 5 batches of organisms harvested at respiratory maxima and another 5 samples obtained at respiratory minima.

sion) occurred when DOT was maximal. Fermentation product concentrations also oscillated at this frequency, but with maxima that show distinct phase relationships (Fig. 1a, b). Thus ethanol increased to maxima at around those of DOT, whereas the acetaldehyde (Fig. $1 \mathrm{~b})$ phase lagged by about $180^{\circ}$. Fig. $1 \mathrm{~b}$ shows the timecourse of accumulation of $\mathrm{H}_{2} \mathrm{~S}$ in the off-gas from the culture during one cycle of respiratory activity. In each cycle, decreasing respiratory activity accompanied the abrupt increase of $\mathrm{H}_{2} \mathrm{~S}$.

Uncoupling of mitochondrial energy conservation leads to increased respiration (Hanstein, 1976). Fig. 2 shows the effect of uncoupling respiration from energy con- 

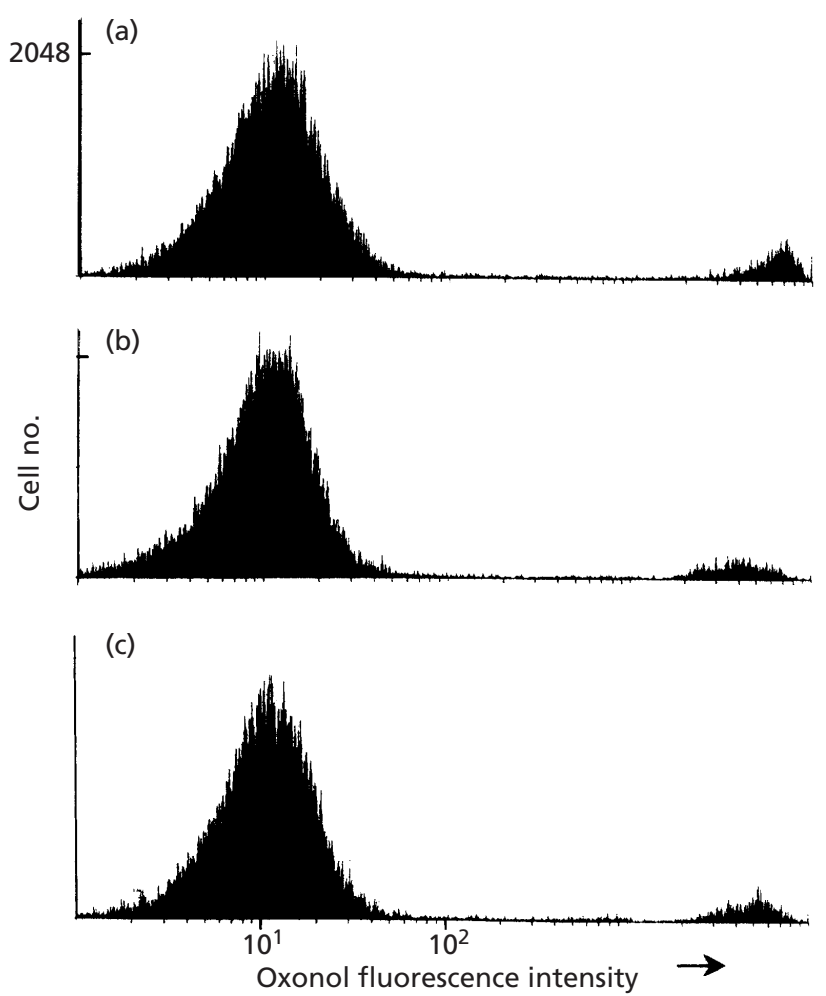

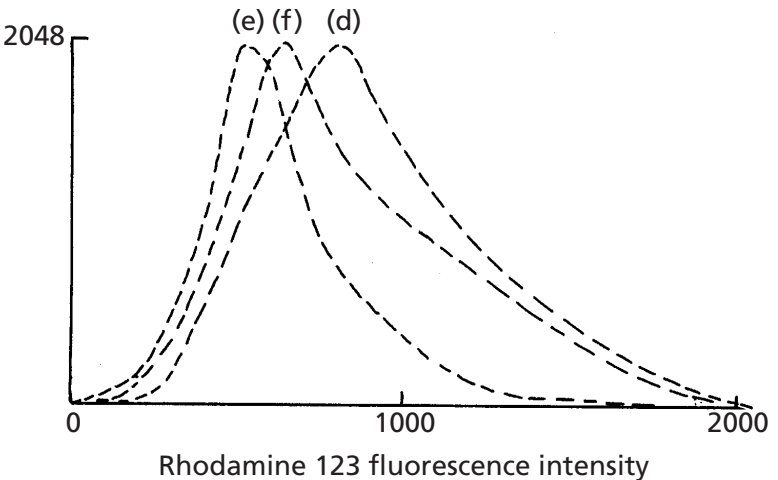

(g)

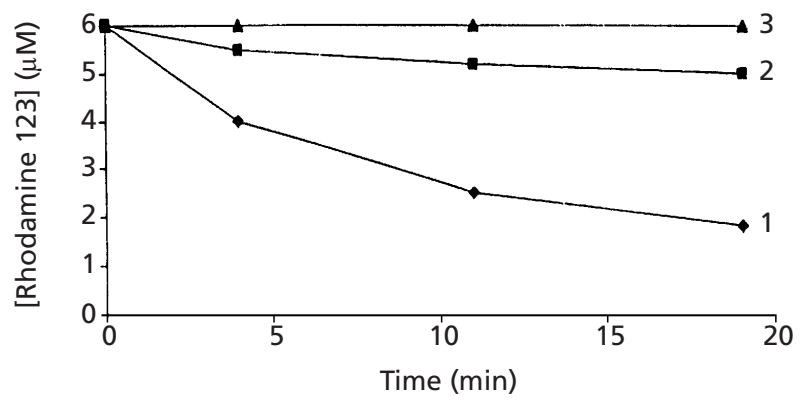

Fig. 4. Flow cytometry of $S$. cerevisiae after incubation with $1 \mu \mathrm{g} \mathrm{ml}^{-1}$ membrane-potential-sensitive fluorophores $\mathrm{DiBAC}_{4}(3)(a-c)$, Rhodamine $123(\mathrm{~d}-\mathrm{f}) ;(\mathrm{a}, \mathrm{c}, \mathrm{d}, \mathrm{f})$ organisms from cultures at respiratory minimum; (b, e) organisms from cultures at a respiratory maximum. (g) Uptake of Rhodamine 123 measured directly into organisms from cultures at respiratory minimum (trace 1 ) and maximum (trace 2). Trace 3, culture sample incubated with $10 \mu \mathrm{M} \mathrm{CCCP.}$

servation in the continuous culture of $S$. cerevisiae. On addition of $10 \mu \mathrm{M}$ CCCP (Fig. 2a) or $4 \mu \mathrm{M} \mathrm{S}-13$ (Fig. 2c), respiration was accelerated; after about $30 \mathrm{~min} \mathrm{O}_{2}$ consumption at both peaks and troughs was increased so that DOT was diminished. During the first cycle after addition of CCCP (Fig. 2b), the period of the oscillation was increased to $60 \mathrm{~min}$; however, after delay times of $11 \mathrm{~h}$ and $4 \mathrm{~h}$ for CCCP (Fig. 2a) and S-13 (Fig. 2c), respectively, successive cycles showed a rapid restoration to the normal period length (48 min). At the

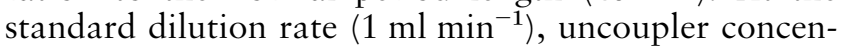
tration was halved in $13 \cdot 3 \mathrm{~h}$. Although uncoupling continued (as indicated by accelerated respiration) oscillatory behaviour recovered.

\section{Sensitivities of yeast populations to inhibitors}

To determine whether the respiratory oscillation could be attributed to changing electron transport pathways or to alterations of the concentrations of fermentation products during continuous culture, samples of organisms were removed, and washed non-proliferating suspensions assayed for respiratory activities. Fig. 3a shows the rates of respiration of washed yeasts sampled at a peak of DOT and tested at the same $\mathrm{pH}$ value as that of the culture $(\mathrm{pH} \mathrm{3.4)}$ in $\mathrm{Na}$ citrate buffer. Glucose addition accelerated respiration. To test whether the electron transport pathway alternative to the main phosphorylating respiratory chain was functional, SHAM, an inhibitor specific for the alternative terminal oxidase (Lloyd \& Edwards, 1978), was employed. SHAM was not very inhibitory $(<10 \%)$; subsequent addition of $5 \mu \mathrm{M} \mathrm{NaN}$ gave $90 \%$ inhibition and $10 \mu \mathrm{M}$ resulted in complete inhibition. When the order of use of these two inhibitors was reversed, similar results were obtained, indicating that respiration was mediated almost entirely by the main azide-sensitive electron transport pathway, and this was so for samples taken both at respiratory maxima and minima (not shown). Organisms still in their culture medium transferred to the closed $\mathrm{O}_{2}$ electrode assay system (Fig. 3b) showed slower $\mathrm{O}_{2}$ consumption than that of washed organisms. Forced aeration for $2 \mathrm{~min}$ (at A and again at B in Fig. 3) gave progressive relief of respiratory inhibition so as to give increased rates of $\mathrm{O}_{2}$ consumption in both types of sample (i.e. those taken from the culture at respiratory troughs or at peaks showed similar results). This suggests that volatile component(s) that have accumulated in the medium during the culture have inhibited respiration. Addition of the distillate (at C and D in Fig. 3) inhibited respiration. These experiments were repeated at least three times with similar results. Fig. 3c 
shows that organisms were not inhibited by $300 \mathrm{mM}$ ethanol. At high concentrations acetaldehyde was inhibitory, but not at those achieved in the cultures. $\mathrm{Na}_{2} \mathrm{~S}$ was very inhibitory even at micromolar concentrations. The protonophore CCCP accelerated respiration, especially with samples from respiratory minima.

\section{Mixing experiments}

To confirm whether accumulation of inhibitor(s) in culture supernatants accounts entirely for changing respiration rate in the continuous culture, cells removed at peak and trough DOT values and then washed, were separately incubated with either type of culture supernatant in the assay system (not shown). Organisms that showed high respiratory rates (harvested at low DOT) continued to utilize $\mathrm{O}_{2}$ rapidly when incubated with culture supernatant from either peaks or troughs of the oscillatory state. Organisms harvested from respiratory troughs (at high DOT) incubated with either type of medium respired slowly. These mixing experiments indicate that fast or slow respiration rates are primarily determined by states intrinsic to the organisms themselves, although the final rates attained by the culture have minor contributions from fermentation products accumulating in the medium.

\section{Changes in membrane potential}

Samples taken at peak and trough DOT values were incubated with $1 \mu \mathrm{g} \mathrm{ml}^{-1}$ of the anionic membranepotential-sensitive dye $\operatorname{DiBAC}_{4}(3)$. The resulting distribution of fluorescence intensities (Fig. $4 \mathrm{a}-\mathrm{c}$ ) indicated an almost homogeneous population ( $>98 \%$ of the total) showing fluorescence emission similar to that due to autofluorescence (not shown). This indicates that the fluorophore had not entered the organisms. The minor, highly fluorescent subpopulation consists of damaged organisms with greatly diminished integrity of the plasma membrane. Organisms from maximal or minimal respiratory phases of the ultradian cycle show identical properties with respect to exclusion of the oxonol dye (median channels of fluorescence intensities at arbitrary value 11 in all three samples).

Incubation with $1 \mu \mathrm{g}$ Rhodamine $123 \mathrm{ml}^{-1}$ indicated diminished dye uptake (Fig. 4d, f) during phases of high respiratory rates (low DOT) at median channels 800 in $\mathrm{d}$ and 600 in $\mathrm{f}$; the fluorescence emission distribution revealed by flow cytometry is indicative of an enhanced inner mitochondrial membrane potential by comparison with that of organisms sampled at low respiration, high DOT (Fig. 4e, median channel at value 450); direct measurement of fluorophore uptake (Fig. 4g) confirmed this. Cell volume determinations, made using a Coulter Channelyser (not shown), indicated that median values were not measurably different in populations harvested at six successive respiratory maxima and minima. Fig. $4 \mathrm{~g}$ shows the uptake rates of Rhodamine 123 into the population of organisms at different stages of the respiratory oscillation.

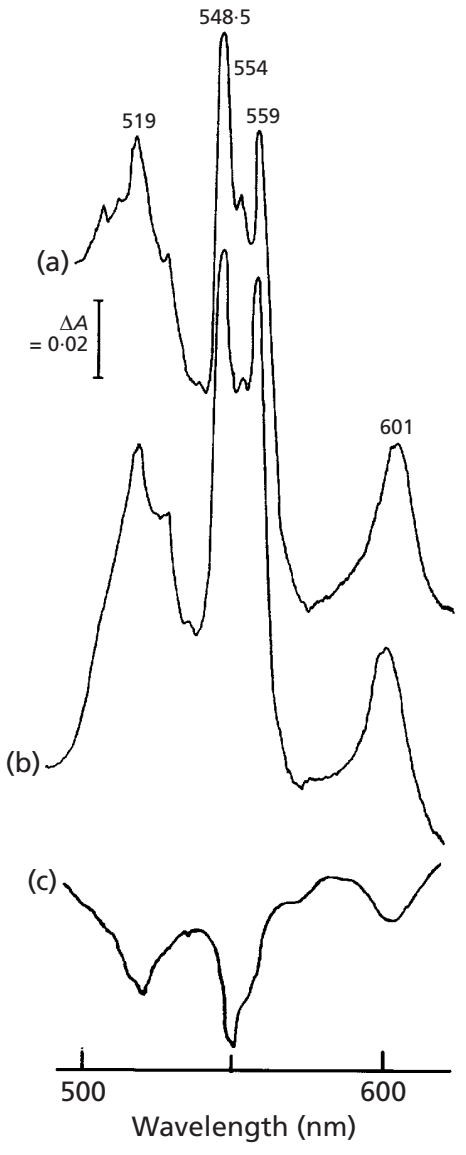

Fig. 5. Low-temperature cytochrome spectra of $S$. cerevisiae at respiratory maxima (a) and minima (b) in a continuous culture. $(a, b)$ Difference spectra: sodium dithionite minus ammonium persulphate oxidized. (c) Direct difference spectra between the continuous culture sampled (at low and high DOT; respiratory maximum minus respiratory minimum).

\section{Cytochromes}

Difference spectra (sodium dithionite reduced minus ammonium persulphate oxidized) indicate that cytochromes $c, c_{1}, b$ and $a+a_{3}$ are not altered in total amounts between the samples taken at respiratory maxima (Fig. 5a) and minima (Fig. 5b). However, when culture samples were rapidly quenched to $77 \mathrm{~K}$ and difference spectra were obtained directly, the resulting absorption maxima (Fig. 5c) indicated that the steadystate redox balances were different between the maxima and minima of respiration. In the samples from cultures at low DOT, cytochromes $c$ and $a+a_{3}$ were more oxidized than in those at high DOT.

\section{Electron microscopy}

At low respiration rates (Fig. 6a) mitochondrial section areas appear large and the cristae were more distinct than those of organisms fixed during the phases of high respiration (Fig. 6b). Other changes also occur; the cell wall structure appeared to be altered in the stage of low respiration. 


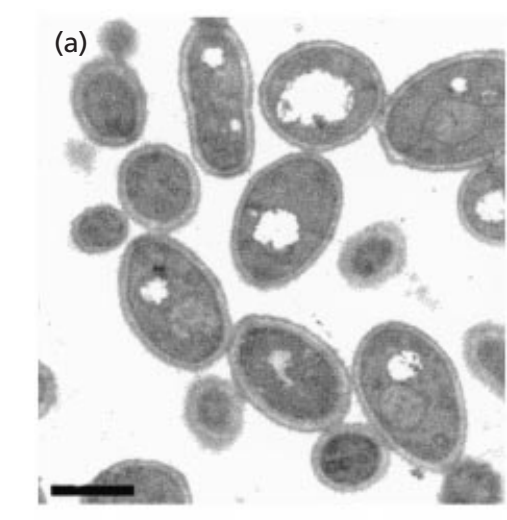

(b)

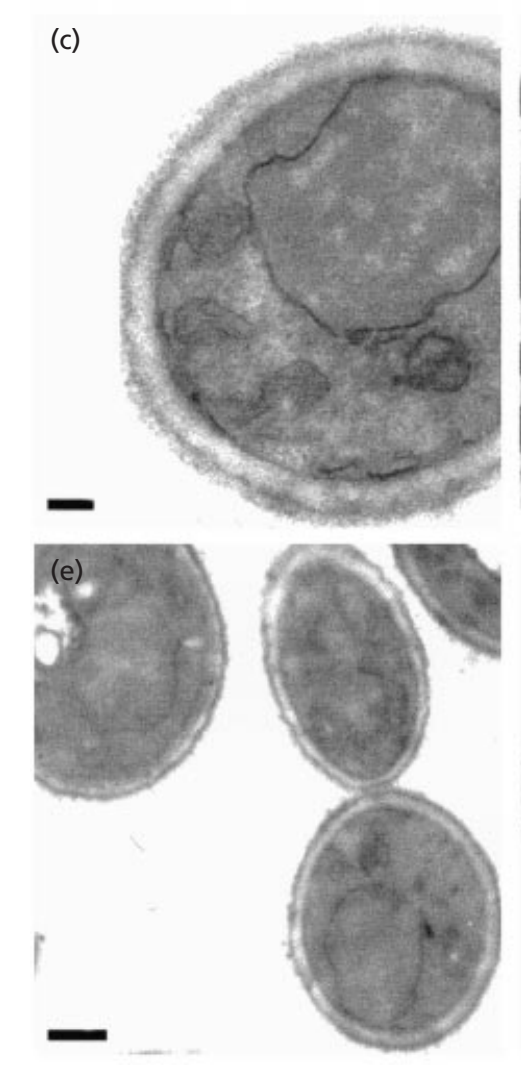

\section{.}

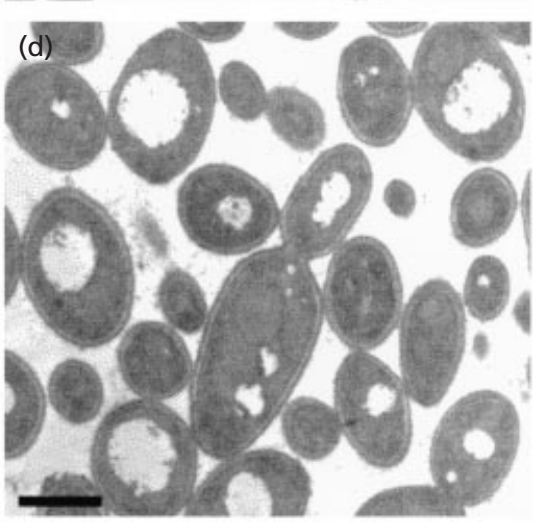

(f)



Fig. 6. Electron micrographs of thin sections of organisms sampled at low DOT (high respiration) $(a-c)$ or high DOT (low respiration) (d-f) states of the continuous culture, respectively. Bars, $2 \mu \mathrm{m}(a, d)$; $0.5 \mu \mathrm{m}(\mathrm{b}, \mathrm{e}) ; 0.2 \mu \mathrm{m}(\mathrm{c}, \mathrm{f})$.

\section{DISCUSSION}

Energy conservation has been studied extensively using 'intact' mitochondria prepared after gentle isolation procedures applied initially to mammalian tissues; subsequently functional mitochondria were isolated from S. cerevisiae (Ohnishi et al., 1966). Irrespective of their source, the respiratory rate of mitochondria in vitro is determined by the presence of a respiratory substrate (e.g. NADH, succinate or malate), $\mathrm{O}_{2}$ and the requirement for oxidative phosphorylation (ADP and $\mathrm{P}_{\mathrm{i}}$, Fig. 7). The respiratory states that correspond to conditions where these various requirements are satisfied were defined in a series of publications (reviewed by Chance \& Williams, 1956). Thus the fully active respiratory system of isolated mitochondria (designated as 'energized' or \#3) may under favourable conditions show a rate of $\mathrm{O}_{2}$ consumption as much as 10 -fold greater than those in the resting or 'de-energized' (\#4) state. When a respiratory substrate, $\mathrm{O}_{2}$ and $\mathrm{P}_{\mathrm{i}}$ are supplied, control of respiration can be demonstrated on making small additions of ADP, as reversible transitions between respiratory states \#3 and \#4 (i.e. increased and decreased $\mathrm{O}_{2}$ consumption rates as measured using a closed $\mathrm{O}_{2}$ electrode system); after the completion of phosphorylation of the added pulse of ADP, the respiratory activity returns to its initial resting (\#4) rate. The effect of protonophore-mediated uncoupling of energy conservation is to increase the respiration of resting mitochondria by short-circuiting the proton translocating ATP synthase and thereby abolishing the dependence of electron transport rates on transmembrane electrochemical potential (Mitchell \& Moyle, 1969). This results in a rapidly respiring uncoupled (state $3 \mathrm{u}$ ) respiratory condition, in which the membrane potential is collapsed. 
State of

Continuous Culture

Low rate of respiration High DOT

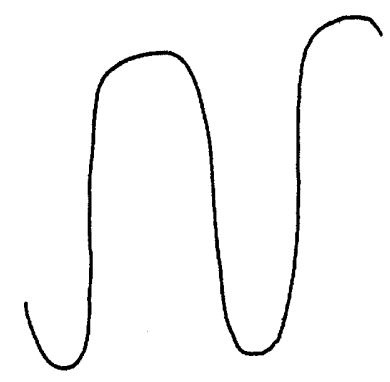

High rate of respiration Low DOT
State of Mitochondria

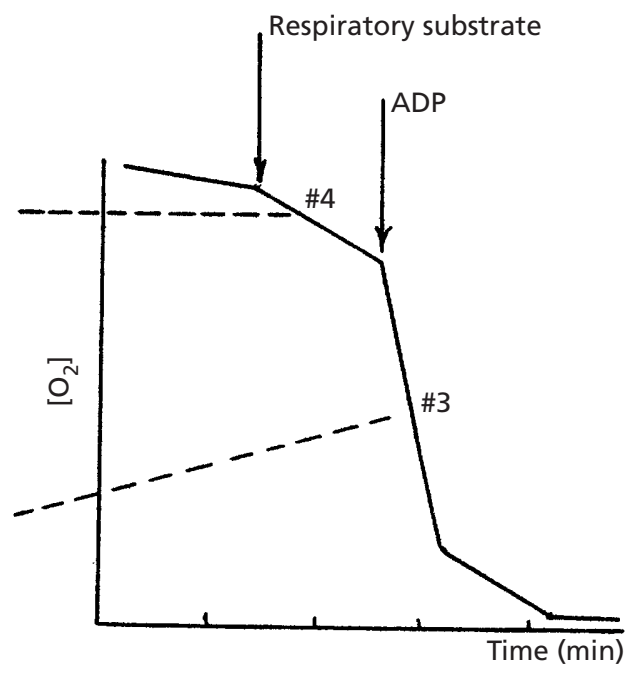

Fig. 7. Changes in mitochondrial structure that accompany altered metabolic status during energy conservation.

These physiological states of mitochondria correspond to distinctive morphological conditions, and the metabolic transitions described above are accompanied by characteristic ultrastructural changes that occur not only in vitro but also in situ (Hackenbrock, 1968a, b). Thus, in resting mitochondria, the cristae are clearly visible, folded into sheets, and most of the internal space is occupied by the matrix. On activation of respiration and oxidative phosphorylation, the intermembrane space expands at the expense of a decrease in the matrix. These conformations are referred to as 'orthodox' and 'condensed' respectively (Fig. 7).

The results presented here strongly suggest that the ultradian-clock-driven oscillatory response of mitochondrial respiration involves continuous cycles of energization and de-energization. There was at any time little detectable flow of electrons through a SHAMsensitive alternative terminal oxidase and thus electron apportionment did not detectably change at any stage of the oscillation. Experiments in which organisms and medium from the two extremes of respiratory activity were mixed confirm that the observed changes are intrinsic to the organisms and do not primarily arise from alteration of medium composition.

Thus during the stage of high DOT (low respiratory activity), mitochondrial transmembrane electrochemical potential is increased, and in section in electron micrographs the mitochondria appear to be in the 'orthodox' conformation (Hackenbrock, 1968a, b). In this state, the organelles are characterized by a relatively large matrix volume and with the inner boundary membrane (the non-cristae component of the inner mitochondrial membrane) closely apposed to the outer membrane, with only a small space between them. In this stage, mitochondrial cytochrome $b$ was more highly reduced, as were the intracellular pools of $\mathrm{NAD}(\mathrm{P}) \mathrm{H}$ and the total flavoprotein.

In the phases of low DOT (high respiratory activity) the mitochondria assume the 'condensed' conformation, in which the inner membrane is pulled away from the outer membrane and the cristae are less clearly defined, the total nicotinamide nucleotide pool is more oxidized as are the cytochromes $c$ and $a+a_{3}$. Thus the succession of events during respiratory oscillation in yeast is similar to that previously described in the soil amoeba Acanthamoeba castellanii (Edwards \& Lloyd, 1978; Lloyd et al., $1982 \mathrm{a}, \mathrm{b})$ where it was concluded that the phenomenon represents in vivo mitochondrial respiratory control (ADP-acceptor control; Chance \& Williams, 1956). The slow kinetics indicates that, as in the amoeba, the cycle of mitochondrial changes in yeast is responding to a slowly changing energetic demand of biosynthetic processes (Edwards \& Lloyd, 1980; Lloyd et al., 1981), i.e. that it is controlled by an ultradian clock (Lloyd \& Edwards, 1984, 1987). That the respiratory oscillation in S. cerevisiae has a temperature-compensated period and therefore has a timekeeping function has recently been shown (Murray et al., 2001) The relaxation time of the oscillating control circuit that determines the $48 \mathrm{~min}$ cycle time lies in a slower time domain than that characteristic of mitochondrial energy metabolism (milliseconds to seconds); it belongs rather in the domain that typically includes the processes of transcription and translation.

Other changes (e.g. the oscillatory accumulation of the fermentation products ethanol, acetaldehyde and $\mathrm{H}_{2} \mathrm{~S}$ ) may contribute to the respiratory oscillation. During glucose-supported growth, the ethanol concentration varied from 110 to $125 \mathrm{mM}$, whereas acetaldehyde ranged from 0.4 to $0.85 \mathrm{mM}$, and $\mathrm{H}_{2} \mathrm{~S}$ varied between 
$1.5 \mu \mathrm{M}$ and an undetectable concentration. Thus the respiratory inhibitor $\mathrm{H}_{2} \mathrm{~S}$ reaches its highest concentration in the culture when respiration is at its lowest. However, $\mathrm{H}_{2} \mathrm{~S}$ never exceeded $1.5 \mu \mathrm{M}$, and at this concentration, inhibition of yeast respiration was less than $10 \%$. Inhibition by acetaldehyde must also occur, but not at the low concentrations $(<0.75 \mathrm{mM})$ monitored during continuous culture $(50 \%$ inhibition requires $100 \mathrm{mM}$ and its maximum accumulation corresponds to maximum respiration).

Unlike the oscillatory respiratory metabolism studied in A. castellanii (Edwards \& Lloyd, 1978, 1980; Lloyd et al., 1982a) or Schizosaccharomyces pombe (Poole et al., 1973), that of Sacch. cerevisiae reported here is not directly coupled to the cell division cycle, i.e. does not require cultures in which the growth and division of the population is synchronized for its detection. Yet there must be synchronization of individual organisms to generate coherence in the populations so as to result in an observable oscillatory behaviour. Rather, in this case there is metabolic coupling between individual cells of the culture. It has been proposed that highly diffusible volatile low molecular weight compound(s), e.g. acetaldehyde and $\mathrm{H}_{2} \mathrm{~S}$, may act as intercellular signalling substances required to maintain coherence of the population (Keulers \&Kuriyama, 1998; Sohn et al., 2000; Sohn \& Kuriyama, 2001). This must be a rapid process (with a time scale of seconds rather than minutes) to coordinate the oscillatory behaviour with such precision (Klevecz \& Murray, 2001).

Further work is required to determine how the oscillation is controlled, i.e. the extramitochondrial events that produce the slow kinetics of transcriptional switching that generate the time-base of the temperaturecompensated ultradian clock (Murray et al., 2001; Salgado et al., 2002). The continuously monitored system described here for study of ultradian clock outputs in $S$. cerevisiae is more convenient and amenable than other ultradian systems (Lloyd, 1992) and many circadian systems (Lloyd et al., 1982b; Lloyd, 1988) where only discrete sampling is possible (Lloyd \& Murray, 2000), and where the accumulation of useful data requires more extended time intervals.

\section{ACKNOWLEDGEMENTS}

D. M. was a holder of a Royal Society STA (Japan) Return Fellowship, L.E. J.S. was financed by Brewing Research International and an Overseas Research Scholarship.

\section{REFERENCES}

Chance, B. \& Williams, R. G. (1956). The respiratory chain and oxidative phosphorylation. Adv Enzymol Relat Areas Mol Biol 17, 65-134.

Chance, B., Legallais, V., Sorge, J. \& Graham, N. (1975). A versatile time-sharing, multichannel spectrophotometer, reflectometer and fluorometer. Anal Biochem 66, 498-514.

Chen, L. B. (1988). Mitochondrial membrane potential in living cells. Annu Rev Cell Biol 4, 155-181.

Dinsdale, M. G., Lloyd, D. \& Jarvis, B. (1995). Yeast vitality during cider fermentation: two approaches to the measurement of membrane potential. J Inst Brew 101, 453-458.

Edwards, S. W. \& Lloyd, D. (1978). Oscillations of respiration and adenine nucleotides in synchronous cultures of Acanthamoeba castellanii. J Gen Microbiol 108, 197-204.

Edwards, S. W. \& Lloyd, D. (1980). Oscillations in protein and RNA content during synchronous growth of Acanthamoeba castellanii. FEBS Lett 109, 21-26.

Hackenbrock, C. R. (1968a). Chemical and physical fixation of isolated mitochondria in low-energy and high-energy states. Proc Natl Acad Sci US A 61, 598-605.

Hackenbrock, C. R. (1968b). Ultrastructural bases for metabolically-linked mechanical activities in mitochondria. II. Electron transport-linked ultrastructural transformation in mitochondria. J Cell Biol 37, 345-369.

Hanstein, W. G. (1976). Uncoupling of oxidative phosphorylation. Biochim Biophys Acta 456, 129-148.

Harrison, D. E. F. \& Chance, B. (1970). Fluorimetric technique for monitoring changes in the level of reduced nicotinamide nucleotides in continuous cultures of microorganisms. Appl Microbiol 19, 446-450.

Keulers, M. \& Kuriyama, H. (1998). Extracellular signalling in oscillatory yeast culture. In Information Processing Cells and Tissues, pp. 85-94. Edited by W. M. L. Holcombe, R. Paton and M. Holcombe. New York: Plenum.

Keulers, M., Suzuki, T., Satroutdinov, A. D. \& Kuriyama, H. (1996a). Autonomous metabolic oscillation in continuous culture of Saccharomyces cerevisiae growth on ethanol. FEMS Microbiol Lett 142, 253-258.

Keulers, M., Satroutdinov, A. D., Suzuki, T. \& Kuriyama, H. (1996b). Synchronisation affector of autonomous short-periodsustained oscillation of Saccharomyces cerevisiae. Yeast 12, 673-682.

Klevecz, R. R. \& Murray, D. B. (2001). Genome wide oscillations in expression. Mol Biol Rep 28, 73-82.

Lloyd, D. (1974). The Mitochondria of Microorganisms. London: Academic Press.

Lloyd, D. (1988). Circadian and ultradian clock-controlled rhythms in unicellular microorganisms. Adv Microb Physiol Biochem 39, 291-298.

Lloyd, D. (1992). Intracellular timekeeping: epigenetic oscillations reveal the functions of an ultradian clock. In Ultradian Rhythms in Life Processes, pp. 5-22. Edited by D. Lloyd and E. R. Rossi. London: Springer.

Lloyd, D. \& Edwards, S. W. (1978). Electron transport pathways alternative to the main phosphorylating chain. In Functions of Alternative Oxidases, pp. 1-10. Edited by H. Degn, D. Lloyd and G. C. Hill. Oxford: Pergamon.

Lloyd, D. \& Edwards, S. W. (1984). Epigenetic oscillations during the cell cycles of lower eukaryotes are coupled to a clock: life's slow dance to the music of time. In Cell Cycle Clocks, pp. 27-46. Edited by L. N. Edmunds. New York: Marcel Dekker.

Lloyd, D. \& Edwards, S. W. (1987). Temperature-compensated ultradian rhythms in lower eukaryotes: timers for cell cycle and circadian events? In Advances in Chronobiology, Part A, pp. 131-151. Edited by J. E. Pauly and L. E. Scheving. New York: Alan R. Liss.

Lloyd, D. \& Murray, D. S. (2000). Redox cycling of intracellular thiols: state variables for ultradian, cell division cycle and circadian cycles. In Redox Behaviour of Circadian Systems, pp. 85-94. Edited by T. Vanden Driessche, J.-L. Guisset and G. P. De Vries. Amsterdam: Kluwer. 
Lloyd, D., Edwards, S. W. \& Williams, J. L. (1981). Oscillatory accumulation of total cellular protein in synchronous cultures of Candida utilis. FEMS Microbiol Lett 12, 295-298.

Lloyd, D., Edwards, S. W. \& Fry, J. C. (1982a). Temperaturecompensated oscillations in respiration and cellular protein content in synchronous cultures of Acanthamoeba castellanii. Proc Natl Acad Sci US A 79, 3785-3788.

Lloyd, D., Poole, R. K. \& Edwards, S. W. (1982b). The Cell Division Cycle: Temporal Control of Cellular Growth and Reproduction. New York: Academic Press.

Luzikov, V. N. (1984). Mechanisms of mitochondrial stabilization. In Mitochondrial Biogenesis and Breakdown, pp. 109-114. New York: Plenum.

Mitchell, P. \& Moyle, J. (1969). Estimation of membrane potential and $\mathrm{pH}$ difference across the cristal membrane of rat-liver mitochondria. Eur J Biochem 7, 471-478.

Murray, D. B., Engelen, F. A., Keulers, M., Kuriyama, H. \& Lloyd, D. (1998a). $\mathrm{NO}^{+}$but not $\mathrm{NO}^{*}$ inhibits respiratory oscillations in ethanol-grown chemostat cultures of Saccharomyces cerevisiae. FEBS Lett 431, 297-299.

Murray, D. B., Engelen, F. A., Keulers, M., Kuriyama, H. \& Lloyd, D. (1998b). $\mathrm{NO}^{+}$but not $\mathrm{NO}^{\circ}$ inhibits respiratory oscillations in ethanol-grown chemostat cultures of Saccharomyces cerevisiae. Trans Biochem Soc 26, S339.

Murray, D. B., Engelen, F., Lloyd, D. \& Kuriyama, H. (1999). Involvement of glutathione in the regulation of respiratory oscillation during a continuous culture of Saccharomyces cerevisiae. Microbiology 145, 2739-2745.

Murray, D. B., Roller, S., Kuriyama, H. \& Lloyd, D. (2001). Clock control of ultradian respiratory oscillation found during yeast continuous culture. J Bacteriol 183, 7253-7259.

Ohnishi, T., Kawaguchi, K. \& Hagihara, B. (1966). Isolation of intact mitochondria from yeast. J Biol Chem 241, 1797-1806.

Poole, R. K., Lloyd, D. \& Kemp, R. B. (1973). Respiratory oscillations and heat evolution in synchronously dividing cultures of the fission yeast Schizosaccharomyces pombe $972 \mathrm{~h}^{-}$.J Gen Microbiol 77, 209-220.

Salgado, L. E. J., Murray, D. B. \& Lloyd, D. (2002). Some antidepressant agents $\left(\mathrm{Li}^{+}\right.$, monoamine oxidase type A inhibitors) perturb the ultradian clock in Saccharomyces cerevisiae. Biol Rhythm Res 33, 351-361.

Satroutdinov, A. D., Kuriyama, H. \& Kobayashi, H. (1992). Oscillatory metabolism of Saccharomyces cerevisiae in continuous culture. FEMS Microbiol Lett 98, 261-268.

Sohn, H.-Y. \& Kuriyama, H. (2001). Ultradian metabolic oscillation of Saccharomyces cerevisiae during aerobic continuous culture: $\mathrm{H}_{2} \mathrm{~S}$, a population synchroniser is produced by sulphite reductase. Yeast 18, 125-135.

Sohn, H.-Y., Murray, D. B. \& Kuriyama, H. (2000). Ultradian oscillation of Saccharomyces cerevisiae during aerobic continuous culture : hydrogen sulphide mediates population synchrony. Yeast 16, 1185-1190.

Vogel, A. I. (1954). Reactions of sulphides. In Macro and Semimicro Qualitative Inorganic Analysis, 4th edn, p. 336. London: Longmans, Green \& Co.

Received 15 April 2002; revised 18 June 2002; accepted 25 June 2002. 